\title{
EQUALIZAÇÃO DE CANAIS DE COMUNICAÇÃO PARA A SINCRONIZAÇÃO DE MAPAS
}

\author{
Renato Candido*, Marcio Eisencraft*, Magno T. M. Silva* \\ * Escola Politécnica, Universidade de São Paulo \\ São Paulo, Brasil
}

Emails: renatocan@lps.usp.br, marcio@lcs.poli.usp.br, magno@lps.usp.br

\begin{abstract}
Many communication systems based on the synchronism of chaotic systems have been proposed in the literature. However, due to the lack of robustness of chaos synchronization, in most cases even minor channel imperfections are enough to hinder communication. In this paper, we propose an adaptive equalization scheme to recover a binary sequence modulated by a chaotic signal, which in turn is generated by an Hénon map. The proposed scheme employs the normalized least-mean-squares (NLMS) algorithm with a modification to enable chaotic synchronization even when the communication channel is not ideal. Simulation results show that the modified NLMS can successfully equalize the channel in different scenarios.
\end{abstract}

Keywords- Dynamical Systems Applications, Chaotic Synchronization, Chaos-based Communication Systems, Adaptive Equalizers.

\begin{abstract}
Resumo- Há vários sistemas de comunicação baseados em sincronismo caótico propostos na literatura. Entretanto, devido à pouca robustez do sincronismo caótico, na maioria dos casos uma pequena imperfeição no canal é suficiente para impedir a comunicação. Neste artigo, é proposto um esquema de equalização adaptativa para recuperar uma sequência binária modulada por um sinal caótico, que por sua vez é gerado por um mapa de Hénon. O esquema proposto utiliza o algoritmo NLMS (Normalized Least-Mean-Squares) com uma modificação para permitir a sincronização caótica mesmo quando o canal de comunicação não é ideal. Os resultados de simulação mostram que o NLMS é capaz de equalizar o canal de comunicação em diferentes cenários.
\end{abstract}

Palavras-chave- Aplicações de Sistemas Dinâmicos, Sincronismo Caótico, Sistemas de Comunicação Baseados em Caos, Equalizadores Adaptativos.

\section{Introdução}

Nas duas últimas décadas, a viabilidade de sistemas de comunicação baseados em sincronismo de sistemas caóticos tem sido investigada tanto teórica como experimentalmente (Pecora e Carroll, 1990; Oppenheim et al., 1992; Cuomo e Oppenheim, 1993; Grzybowski et al., 2011). Um sistema caótico gera, de forma determinística, trajetórias no espaço de estados que são aperiódicas, limitadas e que apresentam dependência sensível às condições iniciais (Alligood et al., 1997). Portanto, sinais caóticos têm sido propostos como portadoras de banda larga para sinais de informação com o potencial de apresentar um alto nível de privacidade na transmissão dos dados (Argyris et al., 2005; Feki et al., 2003).

Recentemente, alguns trabalhos com uma abordagem mais prática usando caos têm surgido principalmente no campo das comunicações óticas (veja, por exemplo, Argyris et al. (2005)). De certa forma, isso é natural já que geradores caóticos podem ser facilmente criados usando as propriedades intrínsecas dos lasers. Este fato foi explorado em Argyris et al. (2005), em que um sistema de comunicação de alta velocidade e longa distância baseado em caos foi demonstrado utilizando-se um enlace comercial de fibra ótica.

Uma das maiores desvantagens dos sistemas de comunicação baseados em caos é a pouca robustez do sincronismo caótico em relação ao nível de ruído e à interferência intersimbólica (ISI
- InterSymbol Interference) introduzidos pelo canal. Mesmo um pequeno nível de ruído ou distorções simples podem ser suficientes para impedir a comunicação (Williams, 2001; Eisencraft et al., 2011; Candido et al., 2013). Assim, a maioria dos trabalhos são baseados na hipótese de utilizar um canal ideal com uma relação sinal ruído alta (veja, por exemplo, Feki et al. (2003), Eisencraft et al. (2009), Grzybowski et al. (2011) e referências contidas). Apesar de alguns resultados preliminares terem sido obtidos em canais mais realísticos (Eisencraft et al., 2013), é de extrema importância que se proponham esquemas que possam se adaptar às imperfeições dos canais de comunicação reais. Caso contrário, esses sistemas não têm muitas chances de chegar a ter interesse prático.

A fim de mitigar a ISI introduzida pelo canal, uma solução usual em sistemas de comunicação convencionais é utilizar um equalizador no receptor (Haykin, 2002). Esquemas de equalização aplicados a sistemas de comunicação baseados em caos têm sido propostos na literatura, utilizando-se diferentes abordagens de codificação da mensagem (veja, por exemplo, Leung (1998), Zhu e Leung (2001), Feng et al. (2004), Ciftci e Williams (2005), Vural e Çetinel (2010), Candido et al. (2013) e referências contidas). Entretanto, somente Candido et al. (2013) consideram a equalização aplicada no domínio de tempo discreto para a modulação caótica que realimenta a sequência transmitida no Gerador de Sinais Caóti- 
cos (GSC). Como essa situação é um bom modelo do experimento descrito por Argyris et al. (2005), esse cenário é considerado relevante e usado neste trabalho. Para gerar os sinais caóticos, é utilizado o mapa de Hénon (Hénon, 1976).

O artigo está organizado da seguinte forma: Na Seção 2, é descrita um versão de tempo discreto de um sistema de comunicação baseado na modulação caótica de Wu e Chua (Eisencraft et al., 2009), que, além de ruído e um canal dispersivo, inclui também um equalizador adaptativo. Um algoritmo do tipo Normalized Least-MeanSquares (NLMS) aplicado à modulação caótica é apresentado na Seção 3. Na Seção 4, é obtido o intervalo do passo de adaptação para garantir a estabilidade local e fraca do algoritmo proposto. Na Seção 5, são apresentados resultados de simulação e na Seção 6, são aprensentadas as conclusões do trabalho.

\section{Formulação do problema}

Na Figura 1, é mostrado o sistema de comunicação considerado, que é uma versão de tempo discreto do sistema proposto por Wu e Chua (Eisencraft et al., 2009). Nesse esquema, o sinal de informação binário $m(n) \in\{-1,+1\}$ é codificado utilizando o primeiro componente do vetor de estados $\mathbf{x}(n)$, i.e.,

$$
s(n)=m(n) x_{1}(n) .
$$

Em seguida, o sinal $s(n)$ é realimentado e transmitido por um canal de comunicação, cujo modelo é constituído por uma função de transferência $H(z)$ e Ruído Gaussiano Branco e Aditivo (AWGN Additive White Gaussian Noise). A fim de mitigar os efeitos do canal, é utilizado um equalizador adaptativo com resposta ao impulso finita com $M$ coeficientes, vetor regressor de entrada $\mathbf{r}(n)=[r(n) r(n-1) \cdots r(n-M+1)]^{T}$ e saída $\widehat{s}(n)=\mathbf{r}^{T}(n) \mathbf{w}(n-1)$, sendo $(\cdot)^{T}$ a operação de transposição e $\mathbf{w}(n-1)$ o vetor de coeficientes do equalizador. O equalizador deve mitigar a interferência intersimbólica introduzida pelo canal e recuperar o sinal codificado $s(n)$ com um atraso de $\Delta$ amostras.

Se o transmissor e o receptor sincronizarem identicamente (Pecora et al., 1997), i.e., se $\widehat{\mathbf{x}}(n) \rightarrow$ $\mathbf{x}(n)$, usando a saída do equalizador e a estimativa de $x_{1}(n)$, o sinal de informação pode ser decodificado por

$$
\widehat{m}(n) \triangleq \widehat{s}(n) / \widehat{x}_{1}(n),
$$

sendo $\widehat{x}_{1}(n)$ o primeiro componente do vetor de estados $\widehat{\mathbf{x}}(n)$. Assume-se que existe uma sequência de treinamento de modo que o erro $e(n)=$ $m(n-\Delta)-\widehat{m}(n)$ seja utilizado para adaptar os coeficientes do equalizador de forma supervisionada.

Neste artigo, o mapa de Hénon (1976) é usado em ambos GSCs na Figura 1. As equações que go- vernam o sistema dinâmico global têm a seguinte forma

$$
\begin{aligned}
& \mathbf{x}(n)=\mathbf{A} \mathbf{x}(n-1)+\mathbf{b}+\mathbf{f}(s(n)), \\
& \widehat{\mathbf{x}}(n)=\mathbf{A} \widehat{\mathbf{x}}(n-1)+\mathbf{b}+\mathbf{f}(\widehat{s}(n)),
\end{aligned}
$$

sendo $\mathbf{x}(n) \triangleq\left[x_{1}(n) \quad x_{2}(n)\right]^{T}, \widehat{\mathbf{x}}(n) \triangleq\left[\begin{array}{ll}\widehat{x}_{1}(n) & \widehat{x}_{2}(n)\end{array}\right]^{T}$.

$$
\mathbf{A}=\left[\begin{array}{ll}
0 & 1 \\
\beta & 0
\end{array}\right], \quad \mathbf{b}=\left[\begin{array}{l}
1 \\
0
\end{array}\right]
$$

e $\mathbf{f}(s(n))=\left[\begin{array}{ll}-\alpha s^{2}(n) & 0\end{array}\right]^{T}$, em que $\alpha$ e $\beta$ parâmetros do mapa. O sistema descrito pela Eq. (3) é autônomo e é chamado de mestre enquanto o sistema descrito pela Eq. (4) depende de $\mathbf{x}(n-1)$ e é chamado de escravo.

Eisencraft et al. (2009) mostraram que, sob condições ideais de canal, i.e., se $r(n)=s(n)$, o sincronismo entre o sistema mestre e o escravo é obtido se todos os autovalores de $\mathbf{A}$ estiverem dentro do círculo unitário no plano complexo. Portanto, como no caso considerado, os autovalores de $\mathbf{A}$ são $\pm \sqrt{\beta}$, conclui-se que, sob condições ideais, ocorre o sincronismo entre mestre e escravo para $|\beta|<1$. Nesse caso, conclui-se das Eqs.(1)(2) que $\widehat{m}(n) \rightarrow m(n)$.

Como $m(n)= \pm 1$, obtém-se a partir da Eq. (1) que $s^{2}(n)=x_{1}^{2}(n)$. Assim, a Eq. (3) não depende de $m(n)$ e a mensagem não interfere nos GSCs com mapas de Hénon. Isso implica que o sinal transmitido é, de fato, caótico contando que os GSCs gerem sinais caóticos. Esta é uma propriedade interessante e não é geral para qualquer GSC ou função de codificação. Por exemplo, utilizando-se uma soma ponderada ao invés de multiplicação na Eq. (1), como é comum na literatura (por exemplo, Wu e Chua (1993) e Eisencraft et al. (2009)), não seria possível garantir que os sinais gerados no GSC continuassem caóticos para qualquer mensagem $m(n)$ ou mesmo que não divergissem.

\section{O algoritmo NLMS caótico}

A fim de se obter um algoritmo do gradiente estocástico para adaptar o equalizador do esquema da Figura 1, é definida a seguinte função-custo instantânea

$$
\hat{J}(n)=e^{2}(n)=[m(n-\Delta)-\widehat{m}(n)]^{2} .
$$

Calculando o gradiente de $\hat{J}(n)$ com relação ao vetor de coeficientes $\mathbf{w}(n-1)$, obtém-se

$$
\begin{aligned}
\boldsymbol{\nabla}_{\mathbf{w}} \hat{J}(n) & =2 e(n) \frac{\partial e(n)}{\partial \mathbf{w}(n-1)} \\
& =-2 e(n) \frac{\partial \widehat{m}(n)}{\partial \mathbf{w}(n-1)}
\end{aligned}
$$




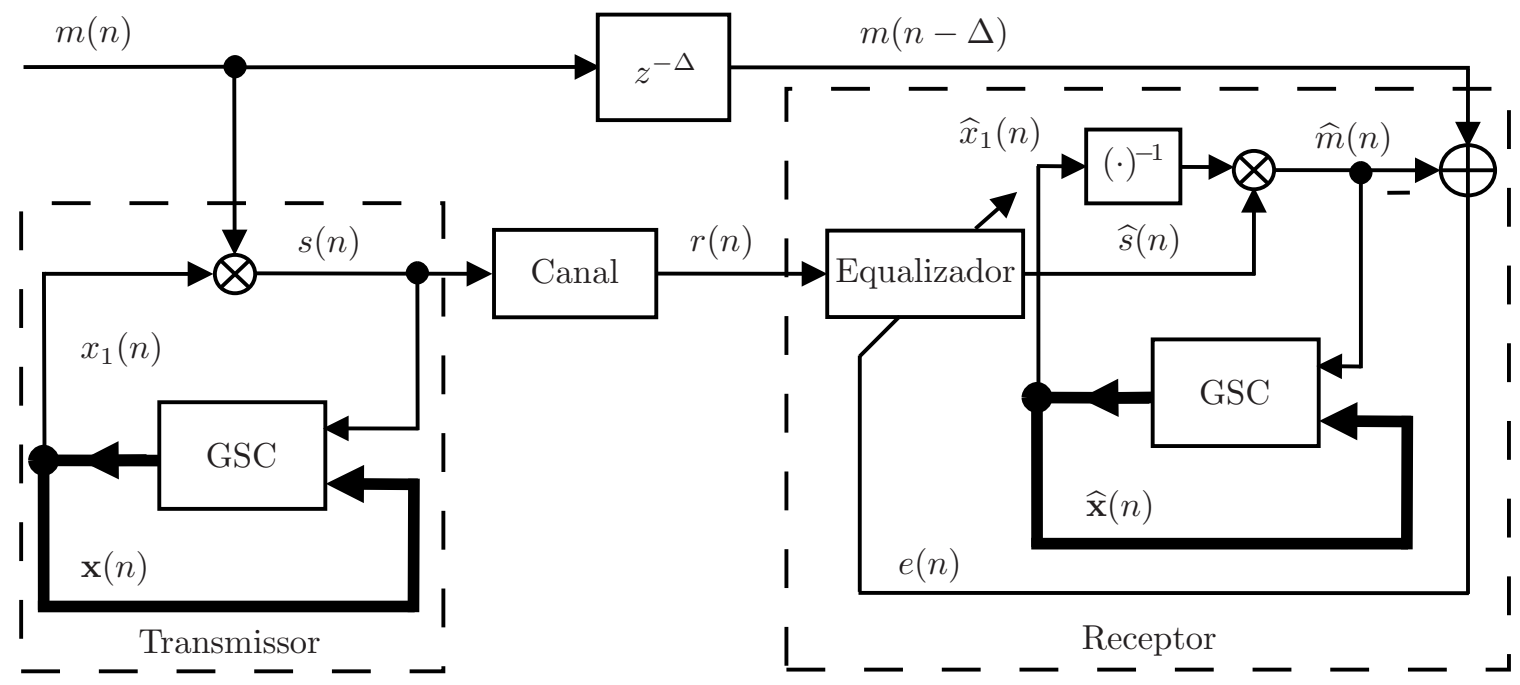

Figura 1: Sistema de comunicação caótico com um equalizador adaptativo.

Considerando-se que $\widehat{x}_{1}(n) \neq 0$ para todo $n$ e levando-se em conta o equalizador no esquema da Figura 1, a Eq. (2) pode ser reescrita como

$$
\widehat{m}(n)=\frac{\widehat{s}(n)}{\widehat{x}_{1}(n)}=\frac{\mathbf{r}^{T}(n) \mathbf{w}(n-1)}{\widehat{x}_{1}(n)} .
$$

Usando-se a Eq. (7) e lembrando-se que $\widehat{x}_{1}(n)$ depende apenas de $\widehat{\mathbf{x}}(n-1)$ e $\widehat{s}(n-1)$, que por sua vez, não depende de $\mathbf{w}(n-1)$, chega-se a

$$
\nabla_{\mathbf{w}} \hat{J}(n)=-2 \frac{e(n)}{\widehat{x}_{1}(n)} \frac{\partial \widehat{s}(n)}{\partial \mathbf{w}(n)}=-2 \frac{e(n)}{\widehat{x}_{1}(n)} \mathbf{r}(n) .
$$

Assim, a equação de adaptação do algoritmo LMS caótico ${ }^{1}$ (cLMS) é dada por

$$
\mathbf{w}(n)=\mathbf{w}(n-1)+\mu \frac{e(n)}{\widehat{x}_{1}(n)} \mathbf{r}(n) .
$$

A fim de se obter uma versão normalizada do cLMS, é definido o erro a posteriori como

$$
e_{p}(n)=m(n-\Delta)-\frac{\mathbf{r}^{T}(n) \mathbf{w}(n)}{\widehat{x}_{1}(n)} .
$$

Usando-se a Eq. (9), $e_{p}(n)$ pode ser reescrito como

$$
\begin{aligned}
e_{p}(n)= & m(n-\Delta) \\
& -\frac{\mathbf{r}^{T}(n)\left[\mathbf{w}(n-1)+\mu \frac{e(n)}{\widehat{x}_{1}(n)} \mathbf{r}(n)\right]}{\widehat{x}_{1}(n)} \\
= & e(n)\left[1-\mu \frac{\|\mathbf{r}(n)\|^{2}}{\widehat{x}_{1}^{2}(n)}\right] .
\end{aligned}
$$

${ }^{1} \mathrm{O}$ termo caótico é usado para os algoritmos apenas para distingui-los das versões originais dos algoritmos LMS e NLMS (veja, por exemplo, Haykin (2002) e Sayed (2008)). A utilização desse termo não implica que os algoritmos tenham um comportamento caótico.
Para se garantir que $e_{p}(n)=0$ a cada iteração $n$, deve-se fazer

$$
\mu(n)=\frac{\widehat{x}_{1}^{2}(n)}{\|\mathbf{r}(n)\|^{2}}
$$

Introduzindo-se um passo de adaptação fixo $\tilde{\mu}$ para controlar a velocidade de convergência e um fator de regularização $\delta$ para evitar a divisão por zero no cálculo de $\mu(n)$ e substituindo-se o passo de adaptação resultante na Eq. (9), obtém-se a equação de atualização do cNLMS, i.e.,

$$
\mathbf{w}(n)=\mathbf{w}(n-1)+\frac{\tilde{\mu}}{\delta+\|\mathbf{r}(n)\|^{2}} \widehat{x}_{1}(n) e(n) \mathbf{r}(n) .
$$

Vale notar que o cNLMS não depende apenas da estimativa do erro $e(n)$ mas também de $\widehat{x}_{1}(n)$. Como $\widehat{x}_{1}(n)$ depende de forma não-linear de $\widehat{s}(n)$, o cNLMS é uma versão não-linear do NLMS. Além disso, o sincronismo entre mestre e escravo no sistema de comunicação caótico depende da eliminação da interferência intersimbólica, que é o papel do equalizador no sistema.

A divisão por zero é evitada no cálculo de $\widehat{m}(n)$ fazendo $\widehat{m}(n)=\operatorname{sign}\left[\widehat{s}(n) \widehat{x}_{1}(n)\right]$ quando $\left|\widehat{x}_{1}(n)\right|<\varepsilon$, sendo $\varepsilon$ uma constante positiva pequena e $\operatorname{sign}[x]=-1$, se $x<0$ ou $\operatorname{sign}[x]=1$, se $x \geq 0$.

Para garantir a estabilidade do algoritmo e evitar estimativas erradas quando $\widehat{x}_{1}(n)$ for muito grande, é introduzido um limite superior para $\widehat{x}_{1}(n)$, i.e., se $\left|\widehat{x}_{1}(n)\right|>X$, impõe-se $\widehat{x}_{1}(n) \leftarrow X \operatorname{sign}\left[\widehat{x}_{1}(n)\right]$, sendo $X$ uma constante positiva. Fazendo $X=100$, não foi observada degradação no desempenho do algoritmo em diversos cenários de simulação.

O algoritmo proposto é mostrado na Tabela 1. 
Tabela 1: Resumo do algoritmo cNLMS.

Inicialize o algoritmo fazendo:

$\mathbf{w}(-1)=\mathbf{0}, \quad \widehat{\mathbf{x}}(0)=[0,1-0,1]^{T}$

$\mathbf{A}=\left[\begin{array}{ll}0 & 1 \\ \beta & 0\end{array}\right], \quad \mathbf{b}=\left[\begin{array}{l}1 \\ 0\end{array}\right], \quad 0<\tilde{\mu}<2$

$\alpha, \beta$ : parâmetros do mapa de Hénon

$\delta, \varepsilon$ : constantes positivas pequenas

$X$ : constante positiva grande

Para $n=0,1,2,3 \ldots$, calcule:

$\widehat{s}(n)=\mathbf{r}^{T}(n) \mathbf{w}(n-1)$

se $\left|\widehat{x}_{1}(n)\right|>X$

$$
\widehat{x}_{1}(n) \leftarrow X \operatorname{sign}\left[\widehat{x}_{1}(n)\right]
$$

fim

se $\left|\widehat{x}_{1}(n)\right| \leq \varepsilon$

$\widehat{m}(n)=\operatorname{sign}\left[\widehat{s}(n) \widehat{x}_{1}(n)\right]$

senão

$$
\widehat{m}(n)=\frac{\widehat{s}(n)}{\widehat{x}_{1}(n)}
$$

fim

$e(n)=m(n-\Delta)-\widehat{m}(n)$

$\mathbf{w}(n)=\mathbf{w}(n-1)+\frac{\widetilde{\mu}_{c}}{\delta+\|\mathbf{r}(n)\|^{2}} \widehat{x}_{1}(n) e(n) \mathbf{r}(n)$

$\widehat{\mathbf{x}}(n+1)=\mathbf{A} \widehat{\mathbf{x}}(n)+\mathbf{b}+\left[\begin{array}{c}-\alpha \widehat{s}^{2}(n) \\ 0\end{array}\right]$

fim

\section{Condições para estabilidade}

Usando a Eq. (7), a equação de adaptação do cNLMS pode ser reescrita como

$$
\begin{aligned}
\mathbf{w}(n)= & {\left[\mathbf{I}-\frac{\widetilde{\mu}}{\delta+\|\mathbf{r}(n)\|^{2}} \mathbf{r}(n) \mathbf{r}^{T}(n)\right] \mathbf{w}(n-1) } \\
& +\widetilde{\mu} \widehat{x}_{1}(n) m(n) \frac{\mathbf{r}(n)}{\delta+\|\mathbf{r}(n)\|^{2}}
\end{aligned}
$$

sendo I a matriz identidade de dimensão $M \times M$. A matriz entre colchetes tem $M-1$ autovalores unitários e um autovalor igual a $\lambda_{1}=1-$ $\widetilde{\mu} \mathbf{r}^{T}(n) \mathbf{r}(n) /\left[\delta+\|\mathbf{r}(n)\|^{2}\right]$.

Notando que

$$
0 \leq \frac{\mathbf{r}^{T}(n) \mathbf{r}(n)}{\delta+\|\mathbf{r}(n)\|^{2}}<1,
$$

e para $\|\mathbf{r}(n)\|^{2} \gg \delta, \mathbf{r}^{T}(n) \mathbf{r}(n) /\left(\delta+\|\mathbf{r}(n)\|^{2}\right) \approx 1$, a fim de se garantir que $\left|\lambda_{1}\right|<1$, deve-se escolher $\widetilde{\mu}$ no intervalo

$$
0<\widetilde{\mu}<2 .
$$

Note-se que a norma da segunda parcela do membro direito da Equação (14) é limitada, i.e.,

$0 \leq \widetilde{\mu}\left|\widehat{x}_{1}(n)\right||m(n)| \frac{\|\mathbf{r}(n)\|}{\delta+\|\mathbf{r}(n)\|^{2}} \leq \widetilde{\mu} X \frac{\sqrt{\delta}}{2 \delta}<\infty$.

Portanto, usando resultados de estabilidade exponencial (determinísticos) do algoritmo LMS
(Sethares, 1992), conclui-se que o cNLMS é estável se $\widetilde{\mu}$ for escolhido no intervalo (15), ou seja, para esses valores de $\widetilde{\mu}$, o algoritmo não diverge (Haykin, 2002).

\section{Resultados de simulação}

Para verificar o comportamento do algoritmo cNLMS foram realizadas simulações considerando o mapa de Hénon com $\alpha=1,4$ e $\beta=0,3$. Os vetores de estados de (3) e (4) foram inicializados como $\mathbf{x}(0)=\mathbf{0}$ e $\widehat{\mathbf{x}}(0)=[0,1-0,1]^{T}$. Outras inicializações também possibilitam bons resultados em termos de sincronismo quando o equalizador é capaz de eliminar razoavelmente bem a interferência intersimbólica. Para as simulações, foi considerada a transmissão de uma sequência binária $m(n) \in\{-1 ; 1\}$ com símbolos equiprováveis e os equalizadores foram inicializados com $\mathbf{w}(0)=\mathbf{0}$. Para efeito de comparação, também foi utilizado o sistema da Figura 1 sem o equalizador, para o qual $\widehat{s}(n)=r(n)$.

Como medida de desempenho, considerou-se o Erro Médio Quadrático em Excesso (EMSE Excess Mean-Square Error) definido como

$$
\mathrm{EMSE} \triangleq \mathrm{E}\left\{e_{a}^{2}(n)\right\},
$$

em que $\mathrm{E}\{\cdot\}$ representa o operador esperança matemática, $e_{a}(n)=\mathbf{r}^{T}(n)\left[\mathbf{w}_{\mathrm{o}}-\mathbf{w}(n-1)\right]$, e $\mathbf{w}_{\mathrm{o}}$ é a solução de Wiener, calculada como $\mathbf{w}_{\mathrm{o}}=\mathbf{R}^{-1} \mathbf{p}$, sendo $\mathbf{R}=\mathrm{E}\left\{\mathbf{r}(n) \mathbf{r}^{T}(n)\right\}$ a matriz de autocorrelação do sinal de entrada do equalizador e $\mathbf{p}=$ $\mathrm{E}\{s(n-\Delta) \mathbf{r}(n)\}$ o vetor de correlação cruzada entre o sinal de entrada e a sequência $s(n-\Delta)$, obtida por meio da modulação da sequência de treinamento (Haykin, 2002; Sayed, 2008).

Num primeiro caso, considerou-se a transmissão da sequência $s(n)$ através do canal com resposta ao impulso infinita

$$
H_{1}(z)=\frac{1}{1+0,6 z^{-1}},
$$

$\operatorname{com} \Delta=0$. A mensagem estimada utilizando-se o equalizador com o algoritmo cNLMS e o erro após a decisão (ambos para uma única realização) são mostrados nas Figuras 2-(b) e (c). A média dos dois coeficientes ao longo das iterações e a curva de EMSE, ambos calculados considerando 1000 realizações, são mostradas nas Figuras 2-(d) e (e). Pode-se observar que o cNMLS converge na média para $\mathbf{w}_{\mathrm{o}} \approx\left[\begin{array}{ll}1 & 0,6\end{array}\right]^{T}$, coeficientes do denominador da função de transferência da Eq. (17), mostrados como linhas tracejadas na Figura 2-(d). Assim, pode-se dizer que o equalizador está funcionando como esperado já que essa solução é capaz de eliminar a interferência intersimbólica, possibilitando a recuperação correta da mensagem transmitida, o que pode ser confirmado pelos erros após a decisão mostrados na Figura 2-(c). Sem a utilização do equalizador, não é possível recuperar a 
mensagem transmitida como pode ser notado na Figura 2-(a)

O efeito do canal também pode ser notado na órbita do sinal transmitido. Na Figura 3-(a), é mostrada a órbita do sinal modulado no transmissor. Com o efeito do canal, as características do sinal transmitido são perdidas, como pode ser notado na Figura 3-(b). Na Figura 3-(c), é possível notar que o equalizador consegue eliminar o efeito do canal, recuperando um sinal com órbita semelhante à do sinal transmitido.
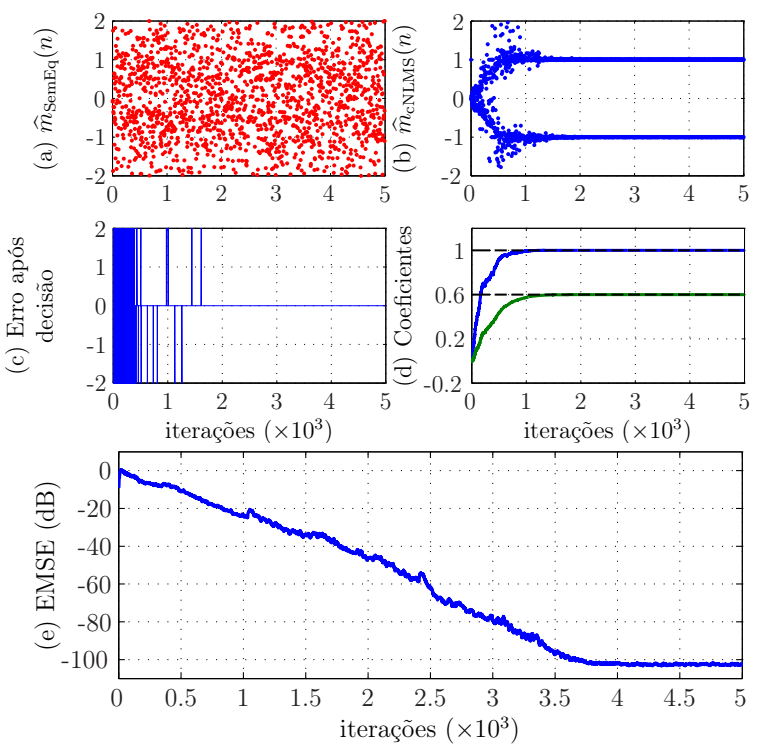

Figura 2: Mensagem recuperada (a) sem equalizador e (b) com cNLMS ( $\left.\mu=0,1, \delta=10^{-2}, \varepsilon=0,1\right)$; (c) Erros após a decisão; (d) Média dos coeficientes do cNLMS e solução de Wiener (linhas tracejadas); (e) EMSE; 1000 realizações; $M=2 ; \Delta=0$.
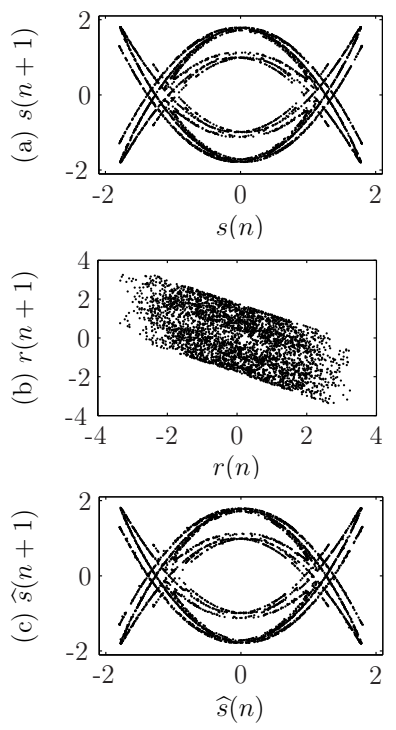

Figura 3: Órbitas dos sinais (a) transmitido, (b) recebido e (c) recuperado no sistema de comunicação da Figura 2
Num segundo caso, considera-se a transmissão da sequência $s(n)$ através do canal (Haykin, 2002)

$$
h(n)= \begin{cases}\frac{1}{2}\left[1+\cos \left(\frac{2 \pi}{W}(n-2)\right)\right], & n=1,2,3 \\ 0, & \text { p.d.v }\end{cases}
$$

Inicialmente, utiliza-se o canal considerando $W=$ 2,9 e, no instante $n=4 \times 10^{3}$, o canal é alterado, fazendo-se $W=3,3$, com $\Delta=7$ em ambos os casos. Os resultados são mostrados na Figura 4. Pode-se notar que o cNLMS converge para a solução ótima de Wiener (Haykin, 2002), cujos coeficientes são mostrados pelas linhas tracejadas na Figura 4-(d). Vale notar que o cNLMS é capaz de de acompanhar a variação abrupta do canal, levando aproximadamente 1000 iterações para atingir o estado estacionário novamente. O equalizador tem um papel importante na eliminação da interferência intersimbólica já que o desempenho do sistema é muito prejudicado quando ele não é utilizado.
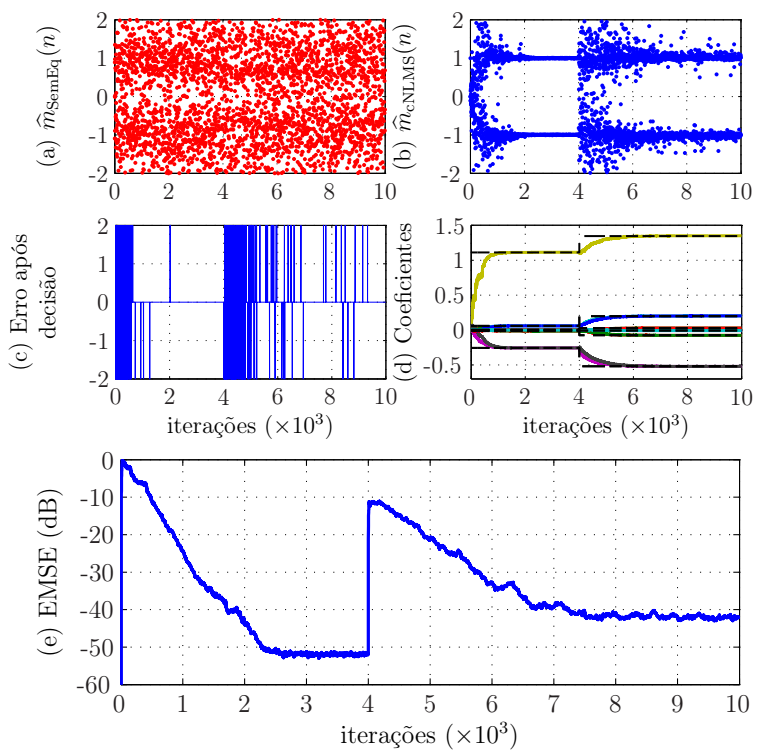

Figura 4: Mensagem recuperada (a) sem equalizador e (b) com cNLMS ( $\left.\mu=0,2, \delta=10^{-5}, \varepsilon=0,1\right)$; (c) Erros após a decisão; (d) Média dos coeficientes do cNLMS e solução de Wiener (linhas tracejadas); (e) EMSE; 1000 realizações; $M=11$; $\Delta=7$.

\section{Conclusões}

Neste trabalho, foi proposto um esquema de equalização supervisionada baseado no algoritmo NLMS para a recuperação de uma mensagem em um sistema de comunicação que utiliza sinais caóticos. Os resultados de simulação indicam que o algoritmo proposto pode permitir a comunicação em sistemas caóticos considerando canais não ideais. Note-se que o esquema proposto não é limitado ao mapa de Hénon. Com modificações adequadas, ele pode ser adequado a qualquer mapa que possa ser escrito na forma da Eq. (3).

Um ponto relevante a ser tratado em trabalhos futuros, é buscar versões autodidatas do es- 
quema de equalização proposto, eliminando-se assim a necessidade de uma sequência de treinamento para que os coeficientes do equalizador convirjam para os valores ótimos.

\section{Agradecimentos}

ME agradece o apoio financeiro do $\mathrm{CNPq}$ (303926/2010-4) e da FAPESP. MTMS também agradece o apoio financeiro do $\mathrm{CNPq}$ (302423/2011-7) e da FAPESP (2012/24835-1).

\section{Referências}

Alligood, K. T., Sauer, T. e Yorke, J. A. (1997). Chaos: An Introduction to Dynamical Systems, Textbooks in Mathematical Sciences, Springer. DOI: 10.1007/978-3-642-59281-2

Argyris, A. et al. (2005). Chaos-based communications at high bit rates using commercial fibre-optic links, Nature 438(7066): 343-346. DOI: $10.1038 /$ nature 04275

Candido, R., Silva, M. T. M. e Eisencraft, M. (2013). Channel equalization for chaotic communications systems, in M. Eisencraft, R. Attux e R. Suyama (eds), Chaotic Signals in Digital Communications, CRC Press, Inc.

Ciftci, M. e Williams, D. (2005). Iterative equalization for chaotic communications systems, Proc. IEEE Int. Conf. Acoustics, Speech, and Signal Process., Vol. 4, pp. 165-68.

Cuomo, K. e Oppenheim, A. (1993). Chaotic signals and systems for communications, Proc. IEEE Int. Conf. Acoustics, Speech, and Sig-nal Process., Vol. 3, pp. 137-140. DOI: 10.1109/ICASSP.1993.319454

Eisencraft, M., Attux, R. R. F. e Suyama, R. (eds) (2013). Chaotic Signals in Digital Communications, CRC Press, Inc.

Eisencraft, M., Fanganiello, R. D. e Baccalá, L. A. (2009). Synchronization of discrete-time chaotic systems in bandlimited channels, Mathematical Problems in Engineering 2009: 1-12. DOI: $10.1155 / 2009 / 207971$

Eisencraft, M., Fanganiello, R. D. e Monteiro, L. H. A. (2011). Chaotic synchronization in discrete-time systems connected by bandlimited channels, IEEE Commun. Lett. 15(6): 671-673. DOI: 10.1109/LCOMM.2011.040111.102309

Feki, M., Robert, B., Gelle, G. e Colas, M. (2003). Secure digital communication using discretetime chaos synchronization, Chaos, Solitons \&. Fractals 18(4): 881-890. DOI: 10.1016/S0960-0779(03)00065-1

Feng, J., C. K. Tse, C. K. e Lau, F. C. M. (2004). Reconstruction of chaotic signals with application to channel equalization in chaos-based communication systems, International Journal of Communication Systems 17: 217-232. DOI: 10.1016/S0960-0779(03)00065-1
Grzybowski, J., Eisencraft, M. e Macau, E. (2011). Chaos-based communication systems: Current trends and challenges, in S. Banerjee, M. Mitra e L. Rondoni (eds), Applications of Chaos and Nonlinear Dynamics in Engineering - Vol. 1, Vol. 71 of Understanding Complex Systems, Springer Berlin / Heidelberg, pp. 203-230.

Haykin, S. (2002). Adaptive Filter Theory, 4th edn, Prentice Hall, Upper Saddle River.

Hénon, M. (1976). A two-dimensional mapping with a strange attractor, Communications in Mathematical Physics 50: 69-77. DOI: 10.1007/BF01608556

Leung, H. (1998). System identification using chaos with application to equalization of a chaotic modulation system, IEEE Trans. Circuits Syst. I 45(3): 314-320. DOI: $10.1109 / 81.662721$

Oppenheim, A. V., Wornell, G. W., Isabelle, S. H. e Cuomo, K. M. (1992). Signal processing in the context of chaotic signals, Proc. IEEE Int. Conf. Acoustics, Speech, and Signal Process., Vol. 4, pp. 117-120.

Pecora, L. M. e Carroll, T. L. (1990). Synchronization in chaotic systems, Phys. Rev. Lett. 64(8): 821-824. DOI: 10.1103/PhysRevLett.64.821

L. M. Pecora, T. L. Carroll, G. A. Johnson, D. J. Mar, and J. F. Heagy, "Fundamentals of synchronization in chaotic systems, concepts, and applications," Chaos: An Interdisciplinary Journal of Nonlinear Science, vol. 7, no. 4, pp. 520-543, 1997.

Sayed, A. H. (2008). Adaptive Filters, Wiley, NJ.

Sethares, W. A. (1992). Adaptive algorithms with nonlinear data and error functions, IEEE Trans. Signal Process. 40(9): 2199-2206.

Vural, C. e Çetinel, G. (2010). Blind equalization of single-input single-output fir channels for chaotic communication systems, Digital Signal Processing 20(1): 201-211. DOI: 10.1016/j.dsp.2009.06.001

Williams, C. (2001). Chaotic communications over radio channels, IEEE Trans. Circuits Syst. I 48(12): 1394 -1404. DOI: 10.1109/TCSI.2001.972846

Wu, C. W. e Chua, L. O. (1993). A simple way to synchronize chaotic systems with applications to secure communication systems, International Journal of Bifurcation and Chaos 3(6): 1619-1627. DOI: $10.1142 / \mathrm{S} 0218127493001288$

Zhu, Z. e Leung, H. (2001). Adaptive blind equalization for chaotic communication systems using extended-Kalman filter, IEEE Trans. Circuits Syst. I 48(8): 979-989. DOI: $10.1109 / 81.940188$ 